Jpn. J. Vet. Sci. 44, 811 814 (1982)

BRIEF NOTE

\title{
Isolation of Mycoplasma hyosynoviae and Other Mycoplasmas from the Respiratory Tracts of Pigs by Aerobic and Anaerobic Cultivation
}

\author{
Seiji KAWAMURA*, Koshi YAMAMOTO and Manabu OGATA \\ Department of Veterinary Microbiology, Faculty of Agriculture, \\ University of Tokyo, 1-1-1 Yayoi, Bunkyo, Tokyo 113
}

(Received 9 November 1981/Accepted 21 May 1982)

Although there have been several reports on mycoplasmal flora of the respiratory tracts of pigs, especially those with enzootic pneumonia $[1,4-6,8,13]$, the incidence and distribution of Mycoplasma hyosynoviae in pigs have been poorly investigated. Our previous report [11] stated that anaerobic cultivation is effective in selective isolation of $M$. hyosynoviae. The present report describes isolation and identification of mycoplasmas other than $M$. hyopneumoniae and $M$. flocculare by anaerobic cultivation from the repiratory tracts of pigs from various herds.

The media used for isolation of mycoplasmas were MAG-Broth and M-Agar as described previously [11]. The pigs examined for mycoplasmas are characterized in Table 1. All pigs except A5 and A6 were derived from herds with enzootic pneumonia. Isolation of mycoplasmas was performed in the same manner as discribed in the previous report [11]. In addition, pneumonic lesion of about $5 \mathrm{~mm}^{3}$ was homogenized with $4 \mathrm{ml}$ of MAG-Broth and the supernatant was used as an inoculum. Identification of isolates was first con- ducted with antisera against $M$. hyorhinis BTS7 and $M$. hyosynoviae S16 by epi-immunofluorescence and in some cases the growth inhibition test and the metabolism inhibition test were also performed. Isolates not identified with these antisera were further tested with those against the following strains: $M$. arginini G230, M. gallinarum PG16, $M$. iners PG30, $M$. salivarium PG20, Acholeplasma laidlawii PG8 and $A$. granularum BTS39.

Distribution of mycoplasmas in the respiratory tracts of seven pigs in group $A$ is given in Table 2. Both $M$. hyorhinis and $M$. hyosynoviae were isolated frequently from the nasal cavities. However, their incidence was lower in the lower respiratory tracts.

The incidence of mycoplasmas in the nasal cavities and pneumonic lungs of pigs from group $\mathrm{A}, \mathrm{B}, \mathrm{C}$ and $\mathrm{D}$ is summarized in Table 3. The overall isolation rates of $M$. hyorhinis and $M$. hyosynoviae from the nasal cavities were very close each other $(70 \%)$, and the rates are almost the same in all the groups examined. Moreover, the coexistence of these two species in the nasal

* Present Address: Department of Animal Pathology, Institute of Medical Science, University of Tokyo, 4-6-1 Shirokanedai, Minato, Tokyo 108. 
Table 1. Pig groups examined

\begin{tabular}{|c|c|c|c|c|c|}
\hline Group & Case No. & $\begin{array}{c}\text { Number of } \\
\text { cases }\end{array}$ & Gross lesion & Sample ${ }^{a}$ & Remark \\
\hline \multirow[t]{2}{*}{ A } & A5-A6 & 2 & None & $N, T, P$ & Experimental animal \\
\hline & $\mathrm{A} 7-\mathrm{A} 11$ & 5 & Pneumonia & $N, T, P L$ & Farm animal \\
\hline \multirow[t]{2}{*}{ B } & BN1-BN17 & 17 & $N I^{b}$ & $N$ & Slaughtered animal \\
\hline & $\begin{array}{l}\text { BL1-BL6 } \\
\text { BL1 10-BL21 }\end{array}$ & 18 & Pneụmonia & $P L$ & Slaughtered animal \\
\hline \multirow[t]{2}{*}{ C } & $\mathrm{CN} 1-\mathrm{CN} 20$ & 20 & $\mathrm{NI}$ & $N$ & Slaughtered animal \\
\hline & $\mathrm{CL} 1-\mathrm{CL}] 8$ & 18 & Pneumonia & $\mathrm{PL}$ & Slaughtered animal \\
\hline $\mathrm{D}$ & D1-D60 & 60 & $\mathrm{NI}$ & $N$ & Slaughtered animal \\
\hline
\end{tabular}

Table 2. Distribution of mycoplasmas in respiratory organs of Group A

\begin{tabular}{|c|c|c|c|c|}
\hline Case No. & Organ $a$ & $\begin{array}{l}\text { M. } \\
\text { hyorhinis }\end{array}$ & $\begin{array}{l}\text { M. } \\
\text { hyosynoviae }\end{array}$ & Others \\
\hline & $N$ & $-{ }^{b}$ & $t^{c}$ & - \\
\hline \multirow[t]{3}{*}{ A5 } & $P$ & - & + & - \\
\hline & $T$ & - & - & - \\
\hline & $N$ & + & + & $+^{d}$ \\
\hline \multirow[t]{3}{*}{ A6 } & $P$ & + & + & - \\
\hline & $T$ & - & - & - \\
\hline & $N$ & - & + & - \\
\hline \multirow[t]{3}{*}{ A7 } & $T$ & - & + & - \\
\hline & $\mathrm{PL}$ & - & - & - \\
\hline & $N$ & + & - & - \\
\hline \multirow[t]{3}{*}{$A 8$} & $T$ & + & - & - \\
\hline & PL & + & - & - \\
\hline & $N$ & + & + & $+^{d}$ \\
\hline \multirow[t]{3}{*}{ A9 } & $T$ & + & + & - \\
\hline & PL & + & - & - \\
\hline & $N$ & + & - & - \\
\hline \multirow[t]{3}{*}{ A 10} & $T$ & + & - & - \\
\hline & $P L$ & - & - & - \\
\hline & $N$ & + & + & - \\
\hline \multirow[t]{3}{*}{ A 11} & $T$ & - & + & - \\
\hline & $\mathrm{PL}$ & - & + & - \\
\hline & $N$ & $5 / 7^{e}$ & $5 / 7$ & $2 / 7$ \\
\hline \multirow[t]{3}{*}{ Total } & $P$ & $1 / 2$ & $2 / 2$ & $0 / 2$ \\
\hline & $T$ & $3 / 7$ & $3 / 7$ & $0 / 7$ \\
\hline & $\mathrm{PL}$ & $2 / 5$ & $1 / 5$ & $0 / 5$ \\
\hline
\end{tabular}

a, N: Nasal cavities. P: Pharynx. T: Trachea. $\mathrm{PL}$ : Pneumonic lungs.

$b$, Negative isolation. $c$, Positive isolation.

a, Acholeplasma laidlawii.

$e$, Number of pigs positive/number of pigs examined. cavities was as high as 50\% throughout the survey. On the contrary, the isolation rates of $M$. hyorhinis and $M$. hyosynoviae from pneumonic lungs were relatively low, and they were about $30 \%$ and $10 \%$, repectively.

Other mycoplasmas were isolated only from the nasal cavities; $A$. laidlawii from nine pigs $(9 \%)$ in three different groups, $M$. salivarium from six pigs $(6 \%)$ in two groups, and $M$. arginini from one pig. The remaining three strains, which were all digitinin-sensitive and arginine-hydrolyzers, were not identified by the antisera against the reference strains used in this study and type strains of following seven species used in the previous study $[11] ; M$. spumans, $M$. opalescens, $M$. gateae, $M$. arthritidis, $M$. gallinarum, $M$. hominis and $M$. orale.

All strains identified as $M$. salivarium were isolated only by anaerobic cultivation and strains of $M$. hyosynoviae and $M$. arginini and three unidentified strains were isolated more easily by anaerobic than by aerobic cultivation. A. laidlawii was isololated by either cultivation.

Relatively high incidence of $M$. hyosynoviae and its frequent coexistence with $M$. hyorhinis in the nasal cavities of pigs in this survey, compared to another report [12], can be attributed partly to the im- 
Table 3. Isolation of mycoplasmas from nasal cavities and pneumonic lungs of Group A-D

\begin{tabular}{|c|c|c|c|c|c|c|c|c|c|c|}
\hline \multirow{2}{*}{ Mycoplasma } & \multicolumn{6}{|c|}{ Positive isolation from nasal cavities } & \multicolumn{4}{|c|}{ Positive isolation from pneumonic lungs } \\
\hline & & $\mathrm{A} / 7^{a}$ & $\mathrm{BN} / 17$ & $\mathrm{CN} / 20$ & $D / 60$ & Total/104 & $\mathrm{A} / 5$ & $\mathrm{BL} / 18$ & $\mathrm{CL} / 18$ & Total $/ 41$ \\
\hline M. hyorhinis & $5^{b}$ & $b(71 \%)$ & $10(59 \%)$ & $17(85 \%)$ & $41(68 \%)$ & $73(70 \%)$ & $2(40 \%)$ & $6(33 \%)$ & $4(22 \%)$ & $12(29 \%)$ \\
\hline M. hyosynoviae & 5 & $(71 \%)$ & $11(65 \%)$ & $14 \quad(70 \%)$ & $41 \quad(68 \%)$ & $71(68 \%)$ & $1(20 \%)$ & $2(11 \%)$ & $1(6 \%)$ & $4(10 \%)$ \\
\hline A. laidlawii & 2 & $(29 \%)$ & $3(18 \%)$ & 0 & $4(7 \%)$ & $9(9 \%)$ & 0 & 0 & 0 & 0 \\
\hline M. salivarium & 0 & & $1(6 \%)$ & 0 & $5(8 \%)$ & $6(6 \%)$ & 0 & 0 & 0 & 0 \\
\hline M. arginini & 0 & & $1(6 \%)$ & 0 & 0 & $1(1 \%)$ & 0 & 0 & 0 & 0 \\
\hline M. spp..$^{c}$ & 0 & & 0 & $2^{d}(10 \%)$ & $j^{e}(2 \%)$ & $3(3 \%)$ & 0 & 0 & 0 & 0 \\
\hline None & 0 & & $2(12 \%)$ & $1(5 \%)$ & $5(8 \%)$ & $8(8 \%)$ & $2(20 \%)$ & $11(61 \%)$ & $13(72 \%)$ & $26(63 \%)$ \\
\hline $\begin{array}{l}\text { Coexistence of } \\
M \text {. hyorhinis } \\
\text { and } \\
M . \text { hyosynoviae }\end{array}$ & 3 & $(43 \%)$ & $7(41 \%)$ & $12(60 \%)$ & $30 \quad\{50 \%\}$ & $52(50 \%)$ & 0 & $1(6 \%)$ & 0 & $1(2 \%)$ \\
\hline
\end{tabular}

$a$, Group/number of cases examined. $b$, Number of positive cases.

$c$, Arginine hydrolysing and digitonin-sensitive strains.

a, Strains CNI6f and CNI7a. e, Lost during identification.

proved isolation of the organism by anaerobic cultivation.

M. salivarium has been regarded as resident flora of human oropharynx [14], and i.olated also from nonhuman primates $[2$, $3,7,9,10]$. Friis [4] reported isolation of a $M$. salivarium-related strain from the nasal mucosa of a pig. In the present survey, $M$. salivarium was isolated from six pigs in two separated groups by anaerobic cultivation, suggesting a prevalence of the organism also among pigs. Further investigation is required to find the distribution of $M$. salivarium among pigs and other animals.

The present study has revealed the usefulness of anaerobic cultivation in isolation of mycoplasmas. Both anaerobic and aerobic cultivation should be used for isolating unspecified mycoplasmas as well as M. hyosynoviae.

\section{REFERENCES}

[ 1] Bannerman, E. S. N., and Nicolet, J. (1971). Schweiz. Arch. Tierheilkd. 113, 697-710.

[2] Cole, B. C., Ward, J. R., Golightly-Rowland, L., and Graham, C. E. (1970). Can. J. Microbiol. 16, 1331-1339.
[3 ] Cole, B. C., Graham, C. E., Colightly-Rowland, L., and Ward, J. R. (1972). Can. J. Microbiol. 18, 1431-1437.

[4] Friis, N. F. (1971). Acta Vet. Scand. 12, 69-79.

[5] Gois, M., Cerny, M., Rozkosny, V., and Sovadina, M. (1969). Zentralbl. Veterinaermed. $B 16,253-265$.

[6] Gois, M., Sisak, F., Kuksa, F., and Sovadina, M. (1975). Zentralbl. Veterinaermed., B22, 205-219.

[7] Hill, A. (1977). Vet. Rec. 101, 117.

[8] L'Ecuyer, C., Switzer, W. P., and Roberts, E. D. (1961). Am. J. Vet. Res. 22, 1020-1025.

[9] Madden, D. L., Hildebrandt, R. J., Monif, G. R. G., London, W. T., McCullough, N. B., and Sever, J. L. (1970a). Lab. Anim. Care 20, 467-470.

[10] Madden, D. L., Hildebrandt, R. J., Monif, G. R. G., London, W. T., McCullough, N. B., and Sever, J. L. (1970b). Lab. Anim. Care 20, 471-473.

[11] Ogata, M., Kawamura, S., and Yamamoto, K. Jpn. J. Vet. Sci. 44, 725-733.

[12] Ross, R. F., and Spear, M. L. (1973). Am. J. Vet. Res. 34, 373-378.

[13] Schulman, A., Estola, T., and Garry-Andersson, A. S. (1970). Zentralbl. Veternaermed. $B 17,549-553$.

[14] Somerson, N. L., and Cole, B. C. (1979). In The Mycoplasmas, Vol. II, Tully, J. G., and Whitcomb, R. F., editors, Academic Press, Inc., New York, 191-216. 
要 約

好気抢よび嫌気培養を用、た豚つ呼吸器からの Mycoplasma hyosynoviae その他のマイコプラズマ の分離 (短報)：河村晴次・山本䓔史・尾形 学 (東京大学農学部家畜微生物学教室) - M. hyosynoviae を主対象として 4 群の豚について呼吸器マイコプラズマの検索を嫌気培養を併用して行った．M. hyorhinis と. M. hyosynoviae は鼻腔に高率（約 70\%）に常在，共存（約 50\%）していたのに対して，両 菌種の肺炎病巣部からの分離率はそれぞれ $30 \% ， 10 \%$ に過ぎなかった。 また，M. salivarium 注鼻腔 から嫌気培養によってのみ分離されたことが注目される。 